\title{
Cigarette smoking in living kidney donors: donor and recipient outcomes
}

\author{
Underwood PW, Sheetz KH, Cron DC, Terjimanian MN, Englesbe \\ MJ, Waits SA. Cigarette smoking in living kidney donors: donor and \\ recipient outcomes.
}

\begin{abstract}
Background: Living kidney donor pools are expanding with the use of "medically complex" donors. Whether or not to include cigarette smokers as living kidney donors remains unclear. The aim of this study was to determine the relationship between donor smoking and recipient outcomes. We hypothesized that donor smoking would increase donor complications and decrease allograft and recipient survival over time.

Methods: The charts of 602 living kidney donors and their recipients were retrospectively reviewed. Kaplan-Meier survival analysis and Cox modeling were used to assess the relationships between smoking and recipient and allograft survival.

Results: No difference in postoperative complications was seen in smoking versus non-smoking donors. Donor smoking at time of evaluation did not significantly decrease allograft survival $(\mathrm{HR}=1.19$, $\mathrm{p}=0.52$ ), but recipient smoking at evaluation did reduce allograft survival $(\mathrm{HR}=1.74, \mathrm{p}=0.05)$. Both donor and recipient smoking decreased recipient survival $(\mathrm{HR}=1.93, \mathrm{p}<0.01$ vs $\mathrm{HR}=1.74$, $\mathrm{p}=0.048)$.

Discussion: When controlled for donor and recipient factors, cigarette smoking by living kidney donors significantly reduced recipient survival. This datum suggests that careful attention to smoking history is an important clinical measure in which to counsel potential donors and recipients. Policy efforts to limit donors with a recent smoking history should be balanced with the overall shortage of appropriate kidney donors.
\end{abstract}

\author{
Patrick W. Underwood, Kyle H. \\ Sheetz, David C. Cron, Michael N. \\ Terjimanian, Michael J. Englesbe \\ and Seth A. Waits
}

Department of Surgery, University of Michigan, Ann Arbor, MI, USA

\author{
Key words: allograft - donor - \\ nephrectomy - outcomes - recipient - \\ smoking - survival \\ Corresponding author: Seth A. Waits, MD, \\ Department of Surgery, University of Michigan, \\ 1500 E Medical Center Dr, Ann Arbor, MI \\ 48109, USA. \\ Tel.: 734615 4028; \\ fax: 7347633187 ; \\ e-mail: waitss@med.umich.edu \\ Conflict of interest: None. \\ Accepted for publication 28 January 2014
}

Amidst a continuing rise in the number of patients on the kidney transplant waitlist, living donor kidney transplantation programs have expanded the criteria for donation. By including elderly and obese donors, centers have boosted the number of acceptable donors and demonstrated satisfactory outcomes (1-3). Despite the resources devoted to studying these "medically complex" donors, little work has been done to assess the risks associated with donor cigarette smoking. As the transplant community continues to expand the donor pool, further investigation of these non-traditional donors is needed.

It remains controversial whether active cigarette smokers should be allowed to donate. A 2007 survey of 132 U.S. kidney transplant programs found that $80 \%$ of programs have a smoking policy when evaluating living donors. Thirty-five percent of programs accept current smokers as living donors, and $36 \%$ require donors to commit to quitting before surgery (4). It is not surprising that the literature surrounding this issue is unclear. A singlecenter study of 100 patients demonstrated that recipients of smoking donors had less improvement in long-term postoperative creatinine and glomerular filtration rate when compared to nonsmoking donors (5). While the recommendation of smoking cessation in kidney transplant recipients is intuitive, inclusion of smokers as potential living donors requires more investigation.

This study is a single-institution retrospective analysis of live donor kidney transplants. We hypothesize that donor smoking will be associated with increased donor complications, decreased recipient allograft survival, and decreased recipient survival. 


\section{Methods}

\section{Study population}

This study was approved by the University of Michigan Institutional Review Board. The charts of patients aged 18 and older who acted as living kidney donors between January 2000 and February 2008 and their recipients were retrospectively reviewed. Age, race, gender, height, weight, BMI, history of smoking, pack-years, and smoking status at evaluation were collected for both donor and recipient. Patients without a record of smoking history in their charts were excluded from the study. Smokeless tobacco and recreational drugs were not included as a positive smoking history. The primary exposure variable was tobacco use at donor or recipient evaluation.

\section{Survival}

Cox modeling was used to assess the relationship between donor smoking status at evaluation and both overall graft recipient survival. The primary outcome for graft survival was time to graft failure. Patients were censored for death with functioning graft or loss of follow-up. For recipient survival, the primary outcome measured was time to death. Patients were censored for earliest time of graft loss or loss of follow-up.

\section{Statistical analysis}

Descriptive statistics were computed for the study cohort. Continuous variables were summarized by mean and standard deviation. Categorical variables were summarized as percentages. A stepwise backward elimination was used on all candidate variables to create a subset of adjustment covariates. All statistical computations were performed in SPSS v17.0 (IBM Corp., Armonk, NY, USA). A two-sided significance level of $\alpha=$ 0.05 was used for analyses.

\section{Results}

\section{Demographics}

Overall, $156(26 \%)$ of the 602 living donors were smoking at the time of evaluation. Overall, there were few clinically significant differences between donors who smoked and those who did not smoke (Table 1). The average age of a kidney donor who was smoking at evaluation was $38.0 \pm 10.2 \mathrm{yr}$ compared with $42.2 \pm 11.1 \mathrm{yr}$ for non-smoking donors $(\mathrm{p}<0.01)$. There were more male smoking donors compared with non-smoking donors
Table 1. Donor and recipient demographics: graft loss and death are reported as unadjusted values

\begin{tabular}{lccr}
\hline Characteristic & $\begin{array}{l}\text { Smoking } \\
\text { donor }\end{array}$ & $\begin{array}{l}\text { Non-smoking } \\
\text { donor }\end{array}$ & p-Value \\
\hline $\mathrm{N}$ & 156 & 446 & \\
Recipient age & $46.0(13.9)$ & $47.4(13.3)$ & 0.27 \\
Recipient sex (male) & $64.7 \%$ & $57.8 \%$ & 0.13 \\
Recipient race (white) & $84.6 \%$ & $82.1 \%$ & 0.47 \\
Recipient height (cm) & $173.0(10.0)$ & $171.8(11.2)$ & 0.21 \\
Recipient weight (kg) & $85.8(20.8)$ & $82.0(21.0)$ & 0.051 \\
Recipient BMl & $28.6(6.2)$ & $27.8(7.6)$ & 0.19 \\
Donor age & $38.0(10.2)$ & $42.2(11.2)$ & $<0.0001$ \\
Donor sex (male) & $46.1 \%$ & $37.2 \%$ & 0.04 \\
Donor race (white) & $83.3 \%$ & $82.5 \%$ & 0.82 \\
Donor height (cm) & $170.7(10.2)$ & $169.3(11.1)$ & 0.15 \\
Donor weight (kg) & $79.4(16.4)$ & $78.6(16.1)$ & 0.60 \\
Donor BMl & $27.2(4.8)$ & $27.5(7.2)$ & 0.56 \\
Recipient graft loss & $23 / 156(14.7)$ & $62 / 446(13.9)$ & 0.06 \\
Recipient death & $46 / 156(29.4)$ & $88 / 446(19.7)$ & $<0.01$ \\
\hline & & &
\end{tabular}

(46.1\% male vs $37.2 \%$ male, $\mathrm{p}=0.04)$. Donor BMI was similar for both smoking donors and non-smoking donors $(27.2 \pm 4.8$ vs $27.5 \pm 7.2$, $\mathrm{p}=0.56)$.

The average age of the recipient was insignificant between smoking donors and non-smoking donors $(46 \pm 13.8$ year vs. $47.4 \pm 13.3$ year, $\mathrm{p}=0.27$ ) Differences between recipient sexes were also insignificant for smoking donors and non-smoking donors (64.7\% male vs 57.8\% male, 0.13$)$. Recipient BMI was similar in smoking donors compared with non-smoking donors $(28.6 \pm 6.2 \mathrm{vs}$ $27.8 \pm 7.6, \mathrm{p}=0.19)$.

\section{Donor complications}

Differences between postoperative complications were insignificant between smoking and nonsmoking donors (Table 2). The most common complication was ileus at $6.73 \%$ in non-smoking donors and $5.13 \%$ in smoking donors. Surgical site infection, urinary retention, and urinary tract infection (UTI) were other common complications with rates under $5 \%$.

Table 2. Donor complications by smoking history

\begin{tabular}{lccc}
\hline Complication & $\begin{array}{l}\text { Smoking } \\
\text { donor }(\%)\end{array}$ & $\begin{array}{l}\text { Non-smoking } \\
\text { donor (\%) }\end{array}$ & p-Value \\
\hline SSI & 4.49 & 2.47 & 0.20 \\
Ileus & 5.13 & 6.73 & 0.50 \\
UTI & 1.92 & 1.57 & 0.77 \\
Urinary retention & 1.92 & 3.81 & 0.26 \\
Hernia & 0 & 0.90 & 0.24 \\
Other & 10.26 & 10.09 & 0.95 \\
\hline
\end{tabular}


Graft survival

Donor smoking did not significantly affect recipient graft survival $(\mathrm{HR}=1.19,95 \% \mathrm{CI}$ : 0.71-1.97, $\mathrm{p}=0.515$; Fig. 1). Allograft recipients who were smokers at the time of evaluation had lower rates of graft survival than recipients who were not smoking at the time of evaluation $(\mathrm{HR}=1.74$, 95\% CI: $1.00-3.04, \mathrm{p}=0.05$ ).

\section{Recipient survival}

Recipients of a kidney from a donor who smoked at the time of evaluation also had lower overall survival rates when compared to recipients who received a kidney from a non-smoking donor $(\mathrm{HR}=1.93,95 \% \mathrm{CI}: 1.27-2.94, \mathrm{p}=0.002$; Fig. 2). Similarly, living kidney donor recipients who smoked at the time of evaluation had significantly lower survival rates compared with recipients who were not smoking at the time of evaluation $(\mathrm{HR}=1.74,95 \% \mathrm{CI}: 1.01-3.00, \mathrm{p}=0.048)$.

\section{Discussion}

A significant proportion of kidney donors have an active smoking history. We know little about how actively smoking at the time of kidney donation affects donor and recipient outcomes. With this study, we compare donor and recipient outcomes from smoking and non-smoking living kidney donors. We demonstrate that donor smoking does not appear to be a significant predictor of graft survival but does appear to have some impact on recipient survival. In addition, complications do not appear to be more prevalent in the donors who smoke. These data can be used as transplant centers consider their living kidney donor smoking policy.

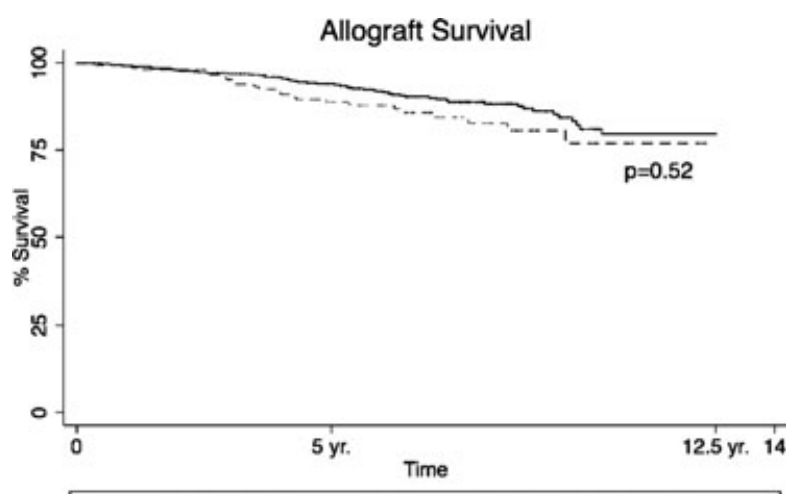

No donor smoking in last year - - - Donor smoking in last yea

Fig. 1. Death-censored graft survival in living donor kidney recipients from smoking and non-smoking donors. There was no significant difference between allograft survival between the two cohorts $(\mathrm{p}=0.52)$.

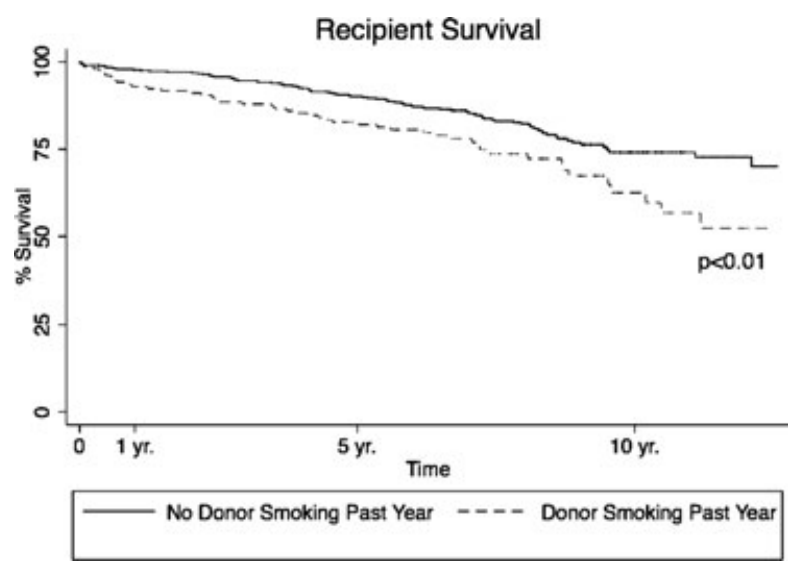

Fig. 2. Recipient survival in living donor kidney recipients from smoking and non-smoking donors. Donor smoking significantly reduced recipient survival $(\mathrm{p}<0.01)$.

Not surprisingly, tobacco smoke has harmful effects on transplanted kidneys. Several studies have investigated the effects of cigarette smoking in kidney transplantation. Recipient smoking has been shown to significantly decrease allograft and recipient survival while cessation prior to transplant can be beneficial (6-8). A large study examining 45307 deceased donor kidney transplants from 1994 to 1999 found a moderate, statistically significant effect on graft and recipient survival (9). The literature surrounding living donor smoking is much less clear. To date, no studies have considered whether living kidney donor smoking affects graft and recipient survival.

As we expand criteria for living donation, we must carefully consider donor risk. Within this context, the modest effect on recipient survival may not provide enough evidence to preclude living kidney donors who smoke. Nonetheless, the transplant community should provide access to resources for smoking cessation. This may help improve the health of both the donor and recipient.

Our analysis has several limitations. As this was a retrospective single-institution study, the generalizability may be limited. Further multi-institutional studies would be required to corroborate these findings. In addition, a more granular smoking history at the time of donation including duration of tobacco use, pack-years, and smoking cessation prior to nephrectomy would be helpful for further elucidation of causal mechanisms. Despite previous work that has isolated donor smoking as a risk factor for graft loss in deceased donors, the quality of allograft from living donors is superior, and the observed effect on allograft survival may be insignificant. As many kidney donors are related to the recipient, second-hand smoking exposure may 
contribute the decreased survival in these recipients. Although a trend toward decreased allograft survival was observed, greater numbers would be needed to reach statistical significance.

The outcomes of this study suggest that transplant providers should be mindful of the risks when evaluating potential living kidney donors who smoke. Donors who are smoking at the time of evaluation should be informed of the increased risks and offered access to smoking cessation resources. Although this paper suggests an association with worse outcomes, more work needs to be done to establish a concrete relationship between living kidney donor smoking and recipient outcomes.

\section{References}

1. Lim WH, Clayton P, Wong G et al. Outcomes of kidney transplantation from older living donors. Transplantation 2013: 95: 106.

2. O'Brien B, Mastoridis S, Sabharwal A, Hakim N, Taube D, Papalois V. Expanding the donor pool: living donor nephrectomy in the elderly and the overweight. Transplantation 2012: 93: 1158 .

3. Heimbach JK, Taler SJ, Prieto M et al. Obesity in living kidney donors: clinical characteristics and outcomes in the era of laparoscopic donor nephrectomy. Am J Transplant 2005: 5: 1057.

4. Rodrigue JR, Pavlakis M, Danovitch GM et al. Evaluating living kidney donors: relationship types, psychosocial criteria, and consent processes at US transplant programs. Am J Transplant 2007: 7: 2326.

5. Heldt J, Torrey R, Han $D$ et al. Donor smoking negatively affects donor and recipient renal function following living donor nephrectomy. Adv Urol 2011: 2011: 929263.

6. Cosio FG, Falkenhain ME, Pesavento TE et al. Patient survival after renal transplantation: II. The impact of smoking. Clin Transplant 1999: 13: 336.

7. Matas AJ, Payne WD, Sutherland DE et al. 2,500 living donor kidney transplants: a single-center experience. Ann Surg 2001: 234: 149 .

8. Sung RS, Althoen M, Howell ta, Ojo AO, Merion RM. Excess risk of renal allograft loss associated with cigarette smoking. Transplantation 2001: 71: 1752.

9. Lin SJ, Koford JK, Baird BC et al. Effect of donors' intravenous drug use, cigarette smoking, and alcohol dependence on kidney transplant outcome. Transplantation 2005: 80: 482 . 